\title{
Association of MMP-9, ADMA, and SCD40L with ischemic stroke and correlations with stroke severity: A case-control study
}

\section{Min Li}

Affiliated Hospital of Chengde Medical College

Hongmei Wang

Affiliated Hospital of Chengde Medical College

Yanjun Gao ( $\sim$ jianchi0370@163.com )

Affiliated Hospital of Chengde Medical College

\section{Research Article}

Keywords: stroke, matrix metalloproteinase-9, asymmetric dimethylarginine, soluble CD40 ligand

Posted Date: August 30th, 2021

DOI: https://doi.org/10.21203/rs.3.rs-821626/v1

License: (c) (i) This work is licensed under a Creative Commons Attribution 4.0 International License.

Read Full License 


\section{Abstract \\ Background}

In the present study, we aimed to examine the correlation of serum levels of MMP-9, ADMA, and SCD40L with the occurrence and severity of acute ischemic stroke.

\section{Methods}

All routines analyses were performed using a Cobas platform. MMP-9, ADMA, and SCD40L were measured using ELISA kit.

\section{Results}

Compared with the controls, the stroke group showed higher MMP-9,higher SCD40L and higher ADMA. There were significant positive correlations between the NIHSS scores and MMP-9, sCD40L and ADMA.

\section{Conclusions}

Our study indicated that serum levels of MMP-9, SCD40L, and ADMA are associated with ischemic stroke and are correlated with the NIHSS scores at admission.

\section{Background}

A stroke is an acute neurological dysfunction episode from either ischemic infarction or a collection of blood within the brain or ventricular system with resultant focal injury of the central nervous system (CNS) [1]. The determination of stroke can be based on clinical evidence of cerebral, spinal cord, or retinal injury based on symptoms or can be made based on pathological, imaging, or other objective evidence of cerebral, spinal cord, or retinal focal injury in a defined vascular distribution [1]. Stroke is a serious public health problem. The estimated global stroke incidence is $2-3$ per 1000 person-years, with older patients and patients with carotid artery stenosis or atrial fibrillation having the highest risk [2,3]. With the westernization of lifestyle habits and the aging of the population, the incidence of cerebrovascular diseases is increasing every year [4-6], especially ischemic stroke, which accounts for $70 \%-80 \%$ of cerebrovascular diseases [1].

Previous studies on various stroke biomarkers have shown that many factors are associated with stroke, including natriuretic peptides, lipoprotein (a), copeptin, procalcitonin, vascular endothelial growth factor, mannose-binding lectin, adipocyte fatty acid-binding protein, inflammatory biomarkers, and cortisol [711]. The optimal biomarkers are still being sought since the available biomarkers display variable sensitivity, specificity, and accuracy. 
Matrix metalloproteinase-9 (MMP-9) can degrade the extracellular matrix to weaken the fibrous cap of atherosclerosis plaques and damage the blood-brain barrier, thus causing brain edema and cerebral hemorrhage transformation $[12,13]$. Thus, high MMP-9 levels appear to be associated with the occurrence and progression of ischemic stroke and prognosis $[12,13]$. Asymmetric dimethylarginine (ADMA) is an endogenous inhibitor of endothelial nitric oxide (NO) synthase (NOS) and is associated with endothelial damage and cardiovascular diseases [14-16]. Soluble CD 40 ligand (SCD40L) is generated by the cleavage of CD40L. High levels of SCD40L can promote platelet activation and aggregation and play an important role in promoting thrombosis and inflammatory reaction [17]. Recent studies have shown a significant increase in plasma SCD40L in acute cerebral infarction patients $[18,19]$. Still, the association of these non-classical factors with stroke remains controversial, and it is poorly known whether they are independent of each other.

Therefore, this study aimed to examine the correlation of serum levels of MMP-9, ADMA, and SCD40L with the occurrence and severity of acute ischemic stroke. The results could help determine the value of these biomarkers for the occurrence of stroke.

\section{Methods}

\section{Study design and patients}

This retrospective study included consecutive patients admitted to the Neurology and Emergency Departments of the Affiliated Hospital of Chengde Medical College from April 2015 to November 2015. This study was approved by the Ethics Committee of the Affiliated Hospital of Chengde Medical College and all methods were performed in accordance with the Helsinki Declaration and relevant guidelines. The requirement for informed consent was waived because of the retrospective nature of the study.

The inclusion criteria were 1) diagnosis of stroke in accordance with the Chinese Guidelines for the Diagnosis and Treatment of Acute Ischemic Stroke (2014) (i. acute onset; ii. focal neurological impairment; iii. imaging lesions or symptoms and signs lasting $>24 \mathrm{~h}$; iv. non-vascular causes were excluded; v. cerebral hemorrhage was excluded by brain CT or MRI) and 2) acute ischemic stroke. The exclusion criteria were 1) cerebral hemorrhage, 2) history of previous stroke, 3) complicated with a serious injury to the heart, liver, kidney, or other organs, 4) chronic infectious diseases, 5) malignant tumors, 6) long-term use of immunosuppressant drugs or hormones before admission, or 7) severe mental disorders.

Control subjects were selected among the patients who visited the Affiliated Hospital of Chengde Medical College for routine health checkups during the same period. The exclusion criteria were 1) history of cerebrovascular disease, 2) complicated with serious damage to the heart, liver, kidney, or other organs, 3) chronic infectious diseases, 4) malignant tumors; 5) long-term use of immunosuppressants or hormones, or 6) severe mental disorders.

\section{Data collection}


Age, sex, smoking history, drinking history, history of hypertension, diabetes, and coronary heart disease, and general laboratory test results at admission (triglycerides, total cholesterol (TC), high-density lipoprotein cholesterol (HDL-C), and low-density lipoprotein cholesterol (LDL-C)) were collected from the medical charts.

\section{Laboratory}

All laboratory measurements were made on fasting peripheral venous blood samples $(5 \mathrm{ml})$ taken within $24 \mathrm{~h}$ of admission, using EDTA tubes. Within 30 min after collection, the plasma was centrifuged for 15 min (3000 rpm) in a high-speed centrifuge, and the plasma was separated. The samples were stored at $-80^{\circ} \mathrm{C}$. All routines analyses were performed using a Cobas platform (Roche Diagnostics, Basel, Switzerland). MMP-9, ADMA, and SCD40L were measured using ELISA kit (Mlbio, Shanghai, China).

\section{Stroke assessment}

All patients admitted for stroke during the study period underwent a routine National Institutes of Health Stroke Scale (NIHSS) scoring.

\section{Statistical analysis}

SPSS 26.0 (IBM, Armonk, NY, USA) was used for statistical analysis. The continuous data were tested for normal distribution using the Kolmogorov Smirnov test. The data conforming to the normal distribution are expressed as means \pm standard deviations and were tested using the independent sample t-test. Data that did not conform to the normal distribution are presented using median (interquartile range) and were compared using the Mann-Whitney U-test. Categorical data are presented as $\mathrm{n}(\%)$ and were tested using the chi-square test. The Spearman correlation analysis was used to analyze the correlations between the indicators. A multivariable logistic regression analysis was used to identify the factors associated with stroke. Two-sided P-values $<0.05$ were considered statistically significant.

\section{Results}

Characteristics of the subjects

A total of 197 subjects who visited the hospital between April 2015 and November 2015 were included. Among them, 135 patients (93 males and 42 females; $59.0 \pm 8.9$ years of age) were admitted due to ischemic stroke, and 62 patients ( 36 males and 26 females; $57.9 \pm 7.4$ years of age) were admitted for a physical. Table 1 presents their characteristics. Hypertension (57.0\% vs. $30.6 \%, P=0.001)$ and smoking $(40.7 \%$ vs. $21.0 \%, P=0.007)$ were more frequent in the stroke group than in controls. Compared with the controls, the stroke group showed higher triglycerides (median, $1.41 \mathrm{vs} .1 .29 \mathrm{mmol} / \mathrm{L}, \mathrm{P}=0.036$ ), lower HDL-C (median, 0.91 vs. 1.06 mmol/L, P = 0.001), higher MMP-9 (median, 323.6 vs. 272.9 mg/L, $\mathrm{P}<$ 0.001 ), higher SCD 40L (median, 588.2 vs. $458.0 \mathrm{mmol} / \mathrm{L}, \mathrm{P}<0.001$ ), and higher ADMA (median, $15.87 \mathrm{vs.}$ $13.86 \mathrm{mmol} / \mathrm{L}, \mathrm{P}<0.001)$. 
Table 1

Patient characteristics

\begin{tabular}{|llll|}
\hline Variables & Control group $(\mathbf{n}=62)$ & Stroke group $(\mathbf{n}=135)$ & $\mathbf{P}$ \\
\hline Age (years) & $57.9 \pm 7.4$ & $59.0 \pm 8.8$ & 0.396 \\
\hline Female, $\mathrm{n}(\%)$ & $26(41.9)$ & $42(31.1)$ & 0.138 \\
\hline Hypertension, $\mathrm{n}(\%)$ & $19(30.6)$ & $77(57.0)$ & 0.001 \\
\hline Coronary heart disease, $\mathrm{n}(\%)$ & $3(4.8)$ & $10(7.4)$ & 0.500 \\
\hline Diabetes mellitus, $\mathrm{n}(\%)$ & $13(21.0)$ & $26(19.3)$ & 0.780 \\
\hline Smoking, $\mathrm{n}(\%)$ & $13(21.0)$ & $55(40.7)$ & 0.007 \\
\hline Drinking, $\mathrm{n}(\%)$ & $14(22.6)$ & $36(26.7)$ & 0.541 \\
\hline Triglycerides (mmol/L) & $1.29(0.82,1.78)$ & $1.41(0.93,2.22)$ & 0.036 \\
\hline TC (mmol/L) & $4.14(3.82,4.7)$ & $4.21(3.62,5.01)$ & 0.750 \\
\hline HDL-C (mmol/L) & $1.06(0.97,1.25)$ & $0.91(0.79,1.09)$ & 0.001 \\
\hline LDL-C (mmol/L) & $2.33(2.04,2.7)$ & $2.18(1.86,2.67)$ & 0.199 \\
\hline MMP-9 (mg/L) & $272.9(135.7,368.3)$ & $323.02(258.6,551.9)$ & $<0.001$ \\
\hline \begin{tabular}{l} 
sCD40L (mmol/L) \\
\hline ADMA (mmol/L)
\end{tabular} & $458.0(367.2,582.6)$ & $588.2(420.6,1337.5)$ & $<0.001$ \\
\hline $\begin{array}{l}\text { TC: total cholesterol; HDL-C: high-density lipoprotein cholesterol; LDL-C: low-density lipoprotein } \\
\text { cholesterol; MMP-9: matrix metalloproteinase-9; sCD 40L: soluble CD40 ligand; ADMA: asymmetric } \\
\text { dimethylarginine. }\end{array}$ & $13.86(8.45,16.95)$ & $15.87(12.50,38.17)$ & $<0.001$ \\
\hline
\end{tabular}

Factors associated with stroke

Table 2 shows that hypertension $(P=0.001)$, smoking $(P=0.008)$, triglycerides $(P=0.010)$, HDL-C $(P=$ 0.031), MMP-9 $(P<0.001), \operatorname{sCD} 40 L(P<0.001)$, and ADMA $(P<0.001)$ were associated with stroke in the univariable analyses. In the multivariable analysis, hypertension $(\mathrm{OR}=2.62,95 \% \mathrm{Cl}: 1.27-5.40, P=0.009)$, triglycerides $(\mathrm{OR}=1.66,95 \% \mathrm{Cl}: 1.08-2.57, \mathrm{P}=0.02), \mathrm{HDL}-\mathrm{C}(\mathrm{OR}=0.36,95 \% \mathrm{Cl}: 0.13-0.98, \mathrm{P}=0.046)$, MMP-9 (OR = 2.30, 95\%Cl: $1.31-4.02, \mathrm{P}=0.004), \operatorname{sCD} 40 \mathrm{~L}(\mathrm{OR}=2.36,95 \% \mathrm{Cl}: 1.26-4.39, \mathrm{P}=0.007)$, and ADMA $(O R=2.02,95 \% \mathrm{Cl}: 1.07-3.83, \mathrm{P}=0.031)$ were independently associated with stroke (Table 2$)$. 
Table 2

Univariable /multivariable regression analysis

\begin{tabular}{|lllll|}
\hline Variable & Univariable & \multicolumn{3}{l|}{ Multivariable } \\
\cline { 2 - 5 } & OR $(95 \% \mathrm{Cl})$ & $\mathbf{P}$ & OR $(95 \% \mathrm{Cl})$ & $\mathbf{P}$ \\
\hline Hypertension & $3.005(1.587-5.689)$ & 0.001 & $2.616(1.268-5.397)$ & 0.009 \\
\hline Smoking & $2.591(1.285-5.225)$ & 0.008 & - & - \\
\hline TG & $1.661(1.128-2.448)$ & 0.010 & $1.664(1.077-2.572)$ & 0.022 \\
\hline HDL-C & $0.38(0.158-0.917)$ & 0.031 & $0.355(0.128-0.984)$ & 0.046 \\
\hline Ln (MMP-9) & $2.737(1.698-4.411)$ & 0.000 & $2.295(1.309-4.024)$ & 0.004 \\
\hline Ln (sCD40L) & $2.936(1.706-5.055)$ & 0.000 & $2.355(1.263-4.391)$ & 0.007 \\
\hline Ln (ADMA) & $2.864(1.669-4.915)$ & 0.000 & $2.022(1.067-3.833)$ & 0.031 \\
\hline $\begin{array}{l}\text { Regression analysis was performed using the natural logarithm transformation of MMP-9, SCD } 40 \text {, } \\
\text { and ADMA. }\end{array}$ & & & \\
\hline $\begin{array}{l}\text { TG: triglycerides; HDL-C: high-density lipoprotein cholesterol; MMP-9: matrix metalloproteinase-9; } \\
\text { sCD40L: soluble CD 40 ligand; ADMA: asymmetric dimethylarginine. }\end{array}$ & \\
\hline
\end{tabular}

Correlation analysis between the NIHSS score on admission and various indicators

The correlation results between the NIHSS score on admission and various indicators were shown in Table 3. The NIHSS scores at admission were not correlated with age, sex, hypertension, coronary heart disease, diabetes mellitus, smoking, drinking, triglycerides, TC, HDL-C, LDL-C, MMP-9, sCD40L, and ADMA. On the other hand, there were significant positive correlations between the NIHSS scores and MMP-9 $(r=$ 0.23, $P=0.007), \operatorname{sCD} 40 L(r=0.37, P<0.001)$, and ADMA $(r=0.26, P=0.003)$ (Table 3 and Fig. 1$)$. 
Table 3

Correlation analysis of the NIHSS score at admission and various indicators

\begin{tabular}{|c|c|c|}
\hline \multirow[t]{2}{*}{ Indicators } & \multicolumn{2}{|l|}{ NIHSS } \\
\hline & $\mathbf{r}$ & $\mathbf{P}$ \\
\hline Female & 0.048 & 0.579 \\
\hline Age & 0.056 & 0.519 \\
\hline Diabetes mellitus & -0.043 & 0.621 \\
\hline Hypertension & -0.031 & 0.722 \\
\hline Coronary heart disease & -0.008 & 0.923 \\
\hline Smoking & 0.089 & 0.305 \\
\hline Drinking & 0.028 & 0.745 \\
\hline TG & -0.023 & 0.788 \\
\hline $\mathrm{TCH}$ & 0.058 & 0.502 \\
\hline HDL-C & 0.051 & 0.554 \\
\hline LDL-C & -0.071 & 0.414 \\
\hline MMP-9 & $0.231^{\star \star}$ & 0.007 \\
\hline sCD40 & $0.371^{\star \star}$ & $<0.001$ \\
\hline ADMA & $0.257^{\star \star}$ & 0.003 \\
\hline \multicolumn{3}{|l|}{ Note: $\star \star P<<0.01$} \\
\hline \multicolumn{3}{|c|}{$\begin{array}{l}\text { NIHSS; National Institutes of Health Stroke Scale; TC: total cholesterol; HDL-C: high-density lipoprotein } \\
\text { cholesterol; LDL-C: low-density lipoprotein cholesterol; MMP-9: matric metalloproteinase-9; SCD 40L: } \\
\text { soluble CD } 40 \text { ligand; ADMA: asymmetric dimethylarginine. }\end{array}$} \\
\hline
\end{tabular}

\section{Discussion}

MMP-9, ADMA, and SCD40L are possibly associated with stroke. This study aimed to examine the correlation of serum levels of MMP-9, ADMA, and SCD40L with the occurrence and severity of acute ischemic stroke. The results showed that the serum levels of MMP-9, SCD40L, and ADMA are associated with ischemic stroke and are correlated with the NIHSS scores at admission.

MMP-9 is involved in the remodeling of the atherosclerosis plaques by digesting the extracellular matrix that constitutes the fibrous cap, making it more prone to rupture, in which case a thrombus will form when the highly thrombotic necrotic core is exposed to the blood [20]. MMP-9 is also involved in the destabilization of the blood-brain barrier, brain edema, and cerebral hemorrhage transformation, which 
can further deteriorate the condition of patients with stroke $[12,13,21]$. Thus, high MMP-9 levels appear to be associated with the occurrence and progression of ischemic stroke and prognosis [12, 13]. A metaanalysis of case-control studies revealed that the MMP-9 levels are directly correlated with stroke volume, stroke severity, and functional outcome [22]. Although stroke volume and functional outcomes were not assessed in the present study, MMP-9 was independently associated with stroke and positively correlated with the NIHSS scores.

NO is a potent vasodilator and antioxidant necessary to the normal functions of the vascular endothelium [23, 24]. Low levels of NO are associated with endothelial dysfunction, which is in itself a risk factor for the initiation and progression of atherosclerosis $[25,26]$. NO is produced by the NOS enzymes, and the endothelial NOS (eNOS) is directly involved in normal endothelial dysfunction [27, 28]. Besides the numerous factors involved in the transcription levels of eNOS [27, 28], ADMA is an endogenous inhibitor of eNOS, and high levels of ADMA are associated with endothelial damage and cardiovascular diseases [14-16]. Chen et al. [29] showed that ADMA is both a marker and a mediator of stroke. Janes et al. [30] showed that ADMA levels are associated with cerebral small vessel disease in asymptomatic patients. A recent review showed that high ADMA levels were associated with carotid atherosclerosis, which is a risk factor for ischemic stroke [31]. Still, some studies suggest that ADMA levels are not associated with stroke [32-34]. In the present study, ADMA levels were independently associated with stroke and correlated with the NIHSS. The discrepancies among studies could be due to several factors, including sample size, selection of the patients and controls, and genetics.

SCD40L is involved in platelet activation and aggregation, which is involved in ischemic stroke pathogenesis when the plaque necrotic core is exposed [17,35]. High levels of SCD40L are also involved in systemic inflammation, which is involved in pro-atherogenic mechanisms [17]. Plasma SCD 40L levels are elevated in patients with acute ischemic stroke, but the causality relationship is unknown $[18,19]$. A study revealed that SCD40L levels were associated with inflammatory markers levels but not with stroke itself [36]. Since the inflammatory markers could not be analyzed in the present study because they were inconsistently measured among the patients, the present study could not determine whether the independent association of SCD40L with stroke was a direct independent association or through inflammation. Still, a study showed that SCD40L levels were associated with stroke severity and outcomes [18], supporting the correlation between SCD40L and the NIHSS score.

Still, the association of these non-classical factors with stroke remains controversial, and it is poorly known whether they are independent of each other. Indeed, several studies examined different panels of biomarkers for their association with stroke, but few studies examined MMP-9, ADMA, and SCD40L within the same study. In the present study, all three markers were each independently associated with stroke. Future studies should examine whether predictive models could be built using these markers.

The NIHSS is a common neurological function score used to determine the prognosis of patients with cerebral infarction at admission [37-39]. The NIHSS can be used to objectively reflect the severity of cerebral infarction and determine patient prognosis [37-39]. Still, it has limitations [40] that could not be 
controlled for in the present study. These limitations could explain, at least in part, why the correlations were weak (all $r<0.4$ ). Another explanation could also be that MMP-9, sCD40L, and ADMA all contribute together to stroke. In addition, other factors could be involved but were not quantified in this study.

This study has limitations. It was a retrospective study limited to the data available in the charts. The sample size was small and did not allow deeper analyses such as receiver operating characteristics analyses and nomograms. Only the NIHSS score could be used to grade the stroke because it is the grading system used in all patients. Many factors that might be associated with stroke were not evaluated in this study.

\section{Conclusions}

In conclusion, the serum levels of MMP-9, SCD40L, and ADMA in patients with ischemic stroke are significantly higher than those in the control group, suggesting that the serum levels of MMP-9, SCD40L, and ADMA are correlated with the occurrence of acute ischemic stroke. In addition, MMP-9, SCD40L, and ADMA are correlated with the NIHSS score. Future studies should examine whether these markers could be used as predictors of the occurrence of acute ischemic stroke.

\section{Abbreviations}

CNS: Central nervous system

MMP-9: Matrix metalloproteinase-9

ADMA: Asymmetric dimethylarginine

NO: Nitric oxide

NOS: Nitric oxide synthase

sCD40L: Soluble CD40 ligand

TC: Total cholesterol

HDL-C: High-density lipoprotein cholesterol

LDL-C: Low-density lipoprotein cholesterol

NIHSS: National Institutes of Health Stroke Scale

eNOS: Endothelial NOS

\section{Declarations}




\section{Ethics approval and consent to participate}

This study was approved by the Ethics Committee of the Affiliated Hospital of Chengde Medical College and all methods were performed in accordance with the Helsinki Declaration and relevant guidelines. The requirement for informed consent was waived because of the retrospective nature of the study.

\section{Consent for publication}

Not applicable.

\section{Availability of data and materials}

Data sharing is not applicable to this article as no datasets were generated or analysed during the current study.

\section{Competing interests}

The authors declare that they have no competing interests

\section{Funding}

None.

\section{Authors' contributions}

ML carried out the studies, participated in collecting data, and drafted the manuscript. HW performed the statistical analysis and participated in its design. YG participated in acquisition, analysis, interpretation of data, and drafted the manuscript. All authors have read and approved the final manuscript.

\section{Acknowledgements}

The authors thank the staff of the Neurology and Emergency Departments of the Affiliated Hospital of Chengde Medical College for their support and cooperation.

\section{References}

1. Sacco RL, Kasner SE, Broderick JP, Caplan LR, Connors JJ, Culebras A, et al. An updated definition of stroke for the 21 st century: a statement for healthcare professionals from the American Heart Association/American Stroke Association. Stroke. 2013;44:2064-89.

2. Collaborators GBDLRoS, Feigin VL, Nguyen G, Cercy K, Johnson CO, Alam T, et al. Global, Regional, and Country-Specific Lifetime Risks of Stroke, 1990 and 2016. N Engl J Med. 2018;379:2429-37. 
3. Feigin VL, Forouzanfar MH, Krishnamurthi R, Mensah GA, Connor M, Bennett DA, et al. Global and regional burden of stroke during 1990-2010: findings from the Global Burden of Disease Study 2010. Lancet. 2014;383:245-54.

4. Wu S, Wu B, Liu M, Chen Z, Wang W, Anderson CS, et al. Stroke in China: advances and challenges in epidemiology, prevention, and management. Lancet Neurol. 2019;18:394-405.

5. Hu S, Cui B, Mlynash M, Zhang X, Mehta KM, Lansberg MG. Stroke epidemiology and stroke policies in China from 1980 to 2017: A systematic review and meta-analysis. Int J Stroke. 2020;15:18-28.

6. Gao Y, Jiang B, Sun H, Ru X, Sun D, Wang L, et al. The burden of stroke in China: Results from a nationwide population-based epidemiological survey. PLoS One. 2018;13:e0208398.

7. Montellano FA, Ungethum K, Ramiro L, Nacu A, Hellwig S, Fluri F, et al. Role of Blood-Based Biomarkers in Ischemic Stroke Prognosis: A Systematic Review. Stroke. 2021;52:543-51.

8. Bhasin A, Srivastava MVP, Vivekanandhan S, Moganty R, Talwar T, Sharma S, et al. Vascular Endothelial Growth Factor as Predictive Biomarker for Stroke Severity and Outcome; An Evaluation of a New Clinical Module in Acute Ischemic Stroke. Neurol India. 2019;67:1280-5.

9. Gandolfi M, Smania N, Vella A, Picelli A, Chirumbolo S. Assessed and Emerging Biomarkers in Stroke and Training-Mediated Stroke Recovery: State of the Art. Neural Plast. 2017;2017:1389475.

10. Ramiro L, Abraira L, Quintana M, Garcia-Rodriguez P, Santamarina E, Alvarez-Sabin J, et al. Blood Biomarkers to Predict Long-Term Mortality after Ischemic Stroke. Life (Basel). 2021;11.

11. Simpkins AN, Janowski M, Oz HS, Roberts J, Bix G, Dore S, et al. Biomarker Application for Precision Medicine in Stroke. Transl Stroke Res. 2020;11:615-27.

12. Wang L, Deng L, Yuan R, Liu J, Li Y, Liu M. Association of Matrix Metalloproteinase 9 and Cellular Fibronectin and Outcome in Acute Ischemic Stroke: A Systematic Review and Meta-Analysis. Front Neurol. 2020;11:523506.

13. Castellanos M, Sobrino T, Millan M, Garcia M, Arenillas J, Nombela F, et al. Serum cellular fibronectin and matrix metalloproteinase- 9 as screening biomarkers for the prediction of parenchymal hematoma after thrombolytic therapy in acute ischemic stroke: a multicenter confirmatory study. Stroke. 2007;38:1855-9.

14. Sibal L, Agarwal SC, Home PD, Boger RH. The Role of Asymmetric Dimethylarginine (ADMA) in Endothelial Dysfunction and Cardiovascular Disease. Curr Cardiol Rev. 2010;6:82-90.

15. Willeit P, Freitag DF, Laukkanen JA, Chowdhury S, Gobin R, Mayr M, et al. Asymmetric dimethylarginine and cardiovascular risk: systematic review and meta-analysis of 22 prospective studies. J Am Heart Assoc. 2015;4:e001833.

16. Zhou S, Zhu Q, Li X, Chen C, Liu J, Ye Y, et al. Asymmetric dimethylarginine and all-cause mortality: a systematic review and meta-analysis. Sci Rep. 2017;7:44692.

17. Yacoub D, Hachem A, Theoret JF, Gillis MA, Mourad W, Merhi Y. Enhanced levels of soluble CD40 ligand exacerbate platelet aggregation and thrombus formation through a CD40-dependent tumor necrosis factor receptor-associated factor-2/Rac1/p38 mitogen-activated protein kinase signaling pathway. Arterioscler Thromb Vasc Biol. 2010;30:2424-33. 
18. Tony AA, Tony EA, Mohammed WS, Kholef EF. Evaluation of plasma levels of neopterin and soluble CD40 ligand in patients with acute ischemic stroke in upper Egypt: can they surrogate the severity and functional outcome? Neuropsychiatr Dis Treat. 2019;15:575-86.

19. Ferro D, Loffredo L, Polimeni L, Fimognari F, Villari P, Pignatelli P, et al. Soluble CD 40 ligand predicts ischemic stroke and myocardial infarction in patients with nonvalvular atrial fibrillation. Arterioscler Thromb Vasc Biol. 2007;27:2763-8.

20. Olejarz W, Lacheta D, Kubiak-Tomaszewska G. Matrix Metalloproteinases as Biomarkers of Atherosclerotic Plaque Instability. Int J Mol Sci. 2020;21.

21. Lakhan SE, Kirchgessner A, Tepper D, Leonard A. Matrix metalloproteinases and blood-brain barrier disruption in acute ischemic stroke. Front Neurol. 2013;4:32.

22. Ramos-Fernandez M, Bellolio MF, Stead LG. Matrix metalloproteinase-9 as a marker for acute ischemic stroke: a systematic review. J Stroke Cerebrovasc Dis. 2011;20:47-54.

23. Tousoulis D, Kampoli AM, Tentolouris C, Papageorgiou N, Stefanadis C. The role of nitric oxide on endothelial function. Curr Vasc Pharmacol. 2012;10:4-18.

24. Moncada S, Higgs EA. Nitric oxide and the vascular endothelium. Handb Exp Pharmacol. 2006:21354.

25. de Haro Miralles J, Martinez-Aguilar E, Florez A, Varela C, Bleda S, Acin F. Nitric oxide: link between endothelial dysfunction and inflammation in patients with peripheral arterial disease of the lower limbs. Interact Cardiovasc Thorac Surg. 2009;9:107-12.

26. Chen JY, Ye ZX, Wang XF, Chang J, Yang MW, Zhong HH, et al. Nitric oxide bioavailability dysfunction involves in atherosclerosis. Biomed Pharmacother. 2018;97:423-8.

27. Heiss C, Rodriguez-Mateos A, Kelm M. Central role of eNOS in the maintenance of endothelial homeostasis. Antioxid Redox Signal. 2015;22:1230-42.

28. Daiber A, Xia N, Steven S, Oelze M, Hanf A, Kroller-Schon S, et al. New Therapeutic Implications of Endothelial Nitric Oxide Synthase (eNOS) Function/Dysfunction in Cardiovascular Disease. Int J Mol Sci. 2019;20.

29. Chen S, Li N, Deb-Chatterji M, Dong Q, Kielstein JT, Weissenborn K, et al. Asymmetric dimethyarginine as marker and mediator in ischemic stroke. Int J Mol Sci. 2012;13:15983-6004.

30. Janes F, Cifu A, Pessa ME, Domenis R, Gigli GL, Sanvilli N, et al. ADMA as a possible marker of endothelial damage. A study in young asymptomatic patients with cerebral small vessel disease. Sci Rep. 2019;9:14207.

31. Qin Z, Tang L, Huang Q, Chen Y, Zhong W, Tang X. A systematic review of the correlation between serum asymmetric dimethylarginine, carotid atherosclerosis and ischemic stroke. Eur J Clin Invest. 2021:e13558.

32. Rueda-Clausen CF, Cordoba-Porras A, Bedoya G, Silva FA, Zarruk JG, Lopez-Jaramillo P, et al. Increased plasma levels of total homocysteine but not asymmetric dimethylarginine in Hispanic subjects with ischemic stroke FREC-VI sub-study. Eur J Neurol. 2012;19:417-25. 
33. Wanby P, Teerlink T, Brudin L, Brattstrom L, Nilsson I, Palmqvist P, et al. Asymmetric dimethylarginine (ADMA) as a risk marker for stroke and TIA in a Swedish population. Atherosclerosis. 2006;185:2717.

34. Khan U, Hassan A, Vallance P, Markus HS. Asymmetric dimethylarginine in cerebral small vessel disease. Stroke. 2007;38:411-3.

35. Bentzon JF, Otsuka F, Virmani R, Falk E. Mechanisms of plaque formation and rupture. Circ Res. 2014;114:1852-66.

36. Jefferis BJ, Whincup PH, Welsh P, Wannamethee SG, Rumley A, Lawlor DA, et al. Prospective study of circulating soluble CD40 ligand concentrations and the incidence of cardiovascular disease in a nested prospective case-control study of older men and women. J Thromb Haemost. 2011;9:1452-9.

37. Chalos V, van der Ende NAM, Lingsma HF, Mulder M, Venema E, Dijkland SA, et al. National Institutes of Health Stroke Scale: An Alternative Primary Outcome Measure for Trials of Acute Treatment for Ischemic Stroke. Stroke. 2020;51:282-90.

38. Farooque U, Lohano AK, Kumar A, Karimi S, Yasmin F, Bollampally VC, et al. Validity of National Institutes of Health Stroke Scale for Severity of Stroke to Predict Mortality Among Patients Presenting With Symptoms of Stroke. Cureus. 2020;12:e10255.

39. Wouters A, Nysten C, Thijs V, Lemmens R. Prediction of Outcome in Patients With Acute Ischemic Stroke Based on Initial Severity and Improvement in the First 24 h. Front Neurol. 2018;9:308.

40. Lyden P. Using the National Institutes of Health Stroke Scale: A Cautionary Tale. Stroke. 2017;48:513-9.

\section{Figures}



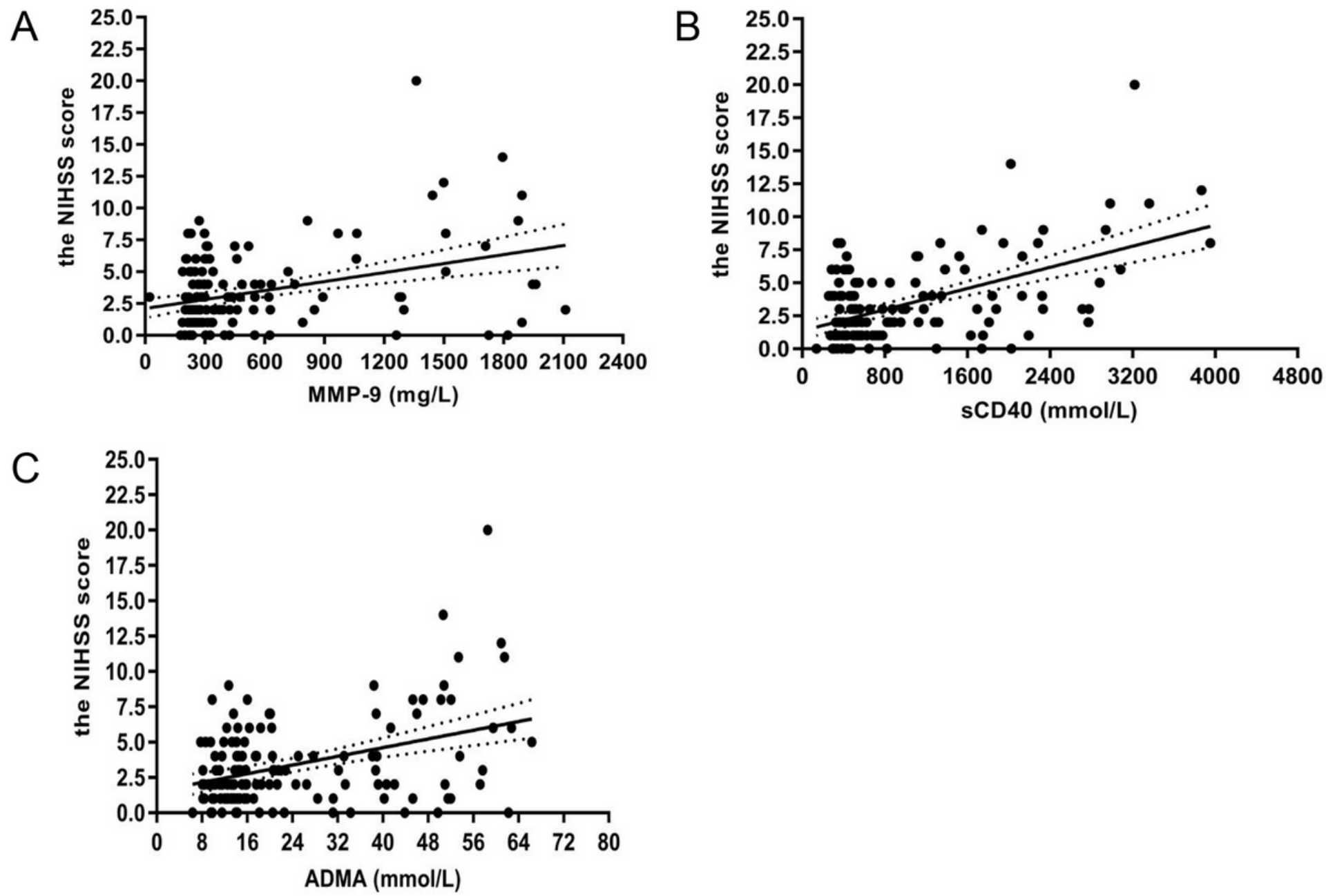

Figure 1

Correlation analysis of the National Institutes of Health Stroke Scale (NIHSS) scores with (A) matrix metalloproteinase-9 (MMP-9), (B) soluble CD40 ligand (sCD40L), and (C) asymmetric dimethylarginine (ADMA). 